\title{
Genetic Fuzzy Approach based Sleep Apnea/Hypopnea Detection
}

\author{
Yashar Maali and Adel Al-Jumaily
}

\begin{abstract}
Sleep Apnea (SA) is one of the most common and important part of sleep disorders. Unfortunately, sleep apnea may be going undiagnosed for years, because of the person's unawareness. The common diagnose procedure usually required an overnight sleep test. During the test, a recording of many biosignals, which related to breath, are obtained by polysomnography machine to detect this syndrome. The manual process for detecting the sleep Apnea by analysis the recording data is highly cost and time consuming. So, several works tried to develop systems that achieve this automatically. This paper proposes a genetic fuzzy approach for detecting Apnea/Hypopnea events by using Air flow, thoracic and abdominal respiratory movement signals and Oxygen desaturation as the inputs. Results show efficiently of this approach.
\end{abstract}

Index Terms-Sleep disorders, genetic fuzzy algorithm, fuzzy sets.

\section{INTRODUCTION}

Study of sleep disorders is important because they are common in general population. For an example, a survey in 1987 [1] reported that at least one symptom of disturbed sleep was present in $41 \%$ of all subjects; and still sleep disorder is common now [2], also it was reported by Young that one daytime sleepiness in 5 adults in 2004 [3].

Sleep disorders have several short term and long term side effects[4]. Short term effect leads to impaired attention and concentration, reduce quality of life, increased rates of absenteeism with reduced productivity, and increased the possibility of accidents at work, home or on the road. Long term consequences of sleep deprivation include increased morbidity and mortality from increasing automobile accidents, coronary artery disease, heart failure, high blood pressure, obesity, type 2 diabetes mellitus, stroke and memory impairment as well as depression.

Sleep Apnea (SA) is one of the most common and important type of sleep disorders, but because of person's unawareness, sleep apnea may go undiagnosed for years [5, 6]. Usually SA case is often observed via patient's spouse or a roommate or a family member who has witnessed the apnea periods alternating with arousals and accompanied by loud snoring $[7,8]$. The patients - whom have symptoms of SA should be examined through an overnight sleep study in sleep center. The diagnosing of SA is usually achieved through using a polysomnography, an integrated device comprising EEG, EMG, EOG, ECG, oxygen saturation [9] airflow

Manuscript received July 15, 2012; revised August 31, 2012.

The authors are with the University of Technology, Sydney Faculty of Engineering and IT Sydney, Australia (Yashar.Maali@student.uts.edu.au; Adel@eng.uts.edu.au). through the mouth and nose, thoracic and abdominal respiration measurement units [10] and the position of the body during sleep. From overnight sleep studies a respiratory disturbance index (RDI) and an Apnea-Hypopnea index (AHI) will be found, the AHI will holds the summation of apneas and hypopneas per hour, while the RDI will holds the summation of apneas, hypopneas and respiratory arousals values. AHI is used to diagnose and grade the severity of the sleep apnea, according to the Chicago criteria: $\mathrm{AHI}<5$, normal; AHI $=5-15$, mild; AHI=15-30, moderate; and AHI $>30$, severe [11].

The polysomnography recording need to be reviewed in order to detect events of SA, the manual review by experts is in fact so cost and time consuming, so several efforts has been done to develop systems that achieve this automatically [12-14]. For this reason several artificial intelligent algorithms are used in this area, like as fuzzy logic that applied to this problem $[13,15,16]$.

This paper presents using of fuzzy inference system, but fuzzy rules are generated by genetic algorithms. Using this approach helps to reach a better accuracy and save time that required establishing the fuzzy rules by interview the specialist, especially when they have different opinions about a same case. The rest of this paper is organized as, pre-processing phase and signal analysis and quantification in section 2, basic of proposed fuzzy inference and genetic algorithm reviewed in section 3 and finally, results and conclusion are presented in section 4 and 5 respectively.

\section{APPROACH AND METHODS}

In this work, events like "fall in airflow" and "desaturation" or "resaturation" in airflow and oxygen saturation, respectively, are desired. Which these events can be defined as follow:

\section{A. Fall in the Air Flow}

Fall or reduction in Airflow (and also thoracic and abdominal respiratory movement signals) defined as follows:

- The mean value of the first minute of the signal is considered as initial value of the normal mean.

- Mean of each specified window of signal (for example each 5 seconds) computed, and temporary classified according with the criteria in Table I.

- If consecutive windows with duration more than 10 s have normal label, the mean value of them will be taken as the new normal mean value.

- Else if, consecutive windows with duration more than 10s represents reduce label, it will be considered as fall in signal and restore start of the fall and percentage of it.

TABLE I: CORRESPONDING LABELS TO EACH LEVEL OF SIGNAL FALLS 


\begin{tabular}{ll}
\hline $\begin{array}{c}\text { Percentage of signal fall } \\
(\boldsymbol{\rho}) \text { with respect to } \\
\text { normal mean }\end{array}$ & Linguistic Label \\
\hline$\rho \leq 25 \%$ & Normal \\
$25 \%<\rho \leq 45 \%$ & Slightly Reduced \\
$45 \%<\rho \leq 70 \%$ & Clearly Reduced \\
$70 \%<\rho \leq 100 \%$ & Totally Reduced \\
\hline
\end{tabular}

\section{B. Oxygen Desaturation}

Blood oxygen desaturation and resaturation are also need to be specified. For this reason, an event in oxygen saturation signal specified as start of fall or start of rise if [13]:

For the start of fall, a point is selected if the fall after that is progressive. In other words, the signal decreases and does not exhibit any significant plateau (i.e. the duration of the plateau should not exceed 30 seconds). The end of fall point is the lowest value obtained following a valid start of fall, as long as the duration of the fall is not greater than $2 \mathrm{~min}$. Once this point has been detected, if the extent of the fall is verified to be at least $4 \%$ and its duration is at least $10 \mathrm{~s}$, then a desaturation has confirmed.

For selecting the start and end points of resaturation it must be considered that, an increase of $3 \%$ should occur in the value of the signal between the start and end points of the rise, and the temporal distance between them should be no greater than $10 \mathrm{~s}$.

In the next step signals are segmented to Reasoning Units (RUs)[16]. Each RU represents an interval which contains at least one event in airflow or oxygen saturation signals.

\section{RUs Algorithm}

The RU algorithm is built based on the following procedure,

1-First, falls in Airflow are determined as explained before.

2- All desaturation witch followed by valid resaturation are determined.

3- Then 30s after each fall in airflow is selected as a RU in the first step.

4- If distance between two consequent RU is greater than length of a RU (30s), one RU with respect to the desaturation events can be made. For this reason, 30s before start of desaturation is selected as a RU in this distance (if possible).

5-Membership functions of each RU are computed regarding to the Table II and Table III[16].

TABLE II: MEMBERSHIP FUNCTION OF DESATURATION

\begin{tabular}{|c|c|c|}
\hline Membership function & Type & $\begin{array}{l}\text { Vector definition } \\
\text { (units in percentage) }\end{array}$ \\
\hline Normal & Trapezoidal & {$\left[\begin{array}{llll}0 & 0 & 1 & 2\end{array}\right]$} \\
\hline Soft & Trapezoidal & {$\left[\begin{array}{lllll}1.8 & 2 & 4 & 5\end{array}\right]$} \\
\hline Strong & Trapezoidal & {$\left[\begin{array}{llll}4 & 6 & 8 & 10\end{array}\right]$} \\
\hline Very Strong & Trapezoidal & {$\left[\begin{array}{llll}9 & 12 & 100 & 100\end{array}\right]$} \\
\hline
\end{tabular}

TABLE III: MEMBERSHIP FUNCTION OF FALL IN AIRFLOW (OR THORACIC AND ABDOMINAL RESPIRATORY MOVEMENT)

\begin{tabular}{|c|c|c|}
\hline Membership function & Type & $\begin{array}{l}\text { Vector definition } \\
\text { (units in percentage) }\end{array}$ \\
\hline Normal & Trapezoidal & {$\left[\begin{array}{llll}0 & 0 & 20 & 30\end{array}\right]$} \\
\hline Soft & Trapezoidal & {$\left[\begin{array}{llll}25 & 35 & 45 & 55\end{array}\right]$} \\
\hline Strong & Trapezoidal & {$\left[\begin{array}{lllll}45 & 60 & 65 & 75\end{array}\right]$} \\
\hline Very Strong & Trapezoidal & {$\left[\begin{array}{llll}70 & 85 & 100 & 100\end{array}\right]$} \\
\hline
\end{tabular}

\section{The Genetic Fuzzy ReAsoning Module}

\section{A. Fuzzy Inference}

DNF (disjunctive normal form) Mamdani fuzzy rule-based systems selected for this study, which has the following general form [17],

$$
\text { If } X_{1} \text { is } \widetilde{A_{1}} \text { and } \ldots \text { and } X_{n} \text { is } \widetilde{A_{n}} \text { THEN } Y \text { is } B \text {, }
$$

where each input variable $X_{i}$ takes a set of linguistic terms $\widetilde{A_{1}}$ as the value, whose members are joined by a disjunctive operator and the output variable remains a usual linguistic variable with a single label associated. Thus, the complete syntax for the antecedent of the rule is

$$
\begin{gathered}
X_{1} \text { is } \widetilde{A_{1}}=\left\{A_{11} \text { or } \ldots \text { or } A_{1 l_{1}}\right\} \text { and } \ldots \\
\text { and } X_{n} \text { is } \widetilde{A_{n}}=\left\{A_{n 1} \text { or } \ldots \text { or } A_{n l_{n}}\right\}
\end{gathered}
$$

An example of this kind of rule is as follows. Let us consider three input as variables, $\mathrm{X}_{1}, \mathrm{X}_{2}$ and $\mathrm{X}_{3}$, and one output variable, $\mathrm{Y}$. Also, each input variable has a linguistic term set named $\mathrm{D}_{\mathrm{i}}$ and linguistic term related to the output is as $\mathrm{F}$,

$$
\begin{gathered}
\widetilde{A_{1}}=\left\{A_{11}, A_{12}, A_{13}\right\}, \widetilde{A_{2}}=\left\{A_{21}, A_{22}, A_{23}, A_{24}\right\} \\
, \widetilde{A_{3}}=\left\{A_{31}, A_{32}, A_{33}\right\}, F=\left\{B_{1}, B_{2}, B_{3}\right\} .
\end{gathered}
$$

In this case, a possible DNF rule may be,

$$
\begin{gathered}
\text { IF } X_{1} \text { is }\left\{A_{11} \text { or } A_{13}\right\} \text { and } X_{2} \text { is }\left\{A_{23} \text { or } A_{24}\right\} \text { and } X_{3} \text { is } \\
\left\{A_{31} \text { or } A_{32}\right\} \text { THEN Y is } B_{2} .
\end{gathered}
$$

In this study 4 inputs which are Oxygen desaturation, Airflow, thoracic and abdominal respiratory movement signals are selected, with membership functions as Table2 and Table3. Also one output, $B_{i}$ as "status", with a singleton membership equal to one is used.

Also, following inference method is selected to compute final output of fuzzy system, $\mathrm{Y}_{0}$

$$
\mathrm{Y}_{0}=\sum_{\mathrm{i}} \mathrm{w}_{\mathrm{i}} \mathrm{h}_{\mathrm{i},} \quad \sum_{\mathrm{i}} \mathrm{w}_{\mathrm{i}}=1,
$$

where $h_{i}$, the output of each rule, is computed as follows,

$$
\mathrm{h}_{\mathrm{i}}=\max \left\{\mathrm{A}_{\mathrm{i}}, \mathrm{B}_{\mathrm{i}}\right\},
$$

and,

$$
\begin{aligned}
& A_{i}=\min \left\{w_{j}{ }^{\prime} A_{j}\right\}, j=1, . .4 \\
& \left(\widetilde{A_{j}}=\max \left\{A_{j 1} \text { or } \ldots \text { or } A_{j l_{j}}\right\}\right) .
\end{aligned}
$$

It must be noted that, $\mathrm{w}$ is the weight related to each rule and $\mathrm{w}^{\prime}$ is the weight of each input signal for the rules.

In this work $\mathrm{Y}_{0}$ is computed related to each $\mathrm{RU}$, then if this value is not less than specified value (singleton defuzzy value), the relevant RU will be selected as sleep Apnea/ Hypopnea event.

\section{B. Genetic Algorithms for Making Fuzzy System}

In this step, genetic algorithm is used for selecting best combination of rules from a set of available rules.

Steps of this algorithm are as follows,

1) Rule generation phase

In this phase, First of all some RUs are selected randomly for training. Then related rules to these RUs are made by the following three steps: 
1-1: One rule related to each RU is made, for example if in selected RU, oxygen saturation is strong Or very strong, Airflow fall is normal, thoracic movement fall is soft Or strong and abdominal respiratory movement fall is normal one rule is made as follow:

$$
\text { If } \begin{aligned}
X_{1} \text { is } \widetilde{A_{1}}=\left\{A_{13} \text { or } A_{14}\right\} \text { and } X_{2} \text { is } \widetilde{A_{2}} \\
=\left\{A_{21}\right\} \text { and } X_{3} \text { is } \widetilde{A_{3}} \\
=\left\{A_{32} \text { or } A_{33}\right\} \text { and }
\end{aligned}
$$

1-2: Also, new rules are made by eliminating one input of the previously made rules in above step. For example ,

$$
\text { If } \begin{aligned}
X_{1} \text { is } \widetilde{A_{1}}=\left\{A_{13}\right. & \text { or } \left.A_{14}\right\} \text { and } X_{3} \text { is } \widetilde{A_{3}} \\
& =\left\{A_{32} \text { or } A_{33}\right\} \text { and } X_{4} \text { is } \widetilde{A_{4}} \\
& =\left\{A_{41}\right\} \text { THEN status is event. }
\end{aligned}
$$

In above example input $X_{2}$ eliminated. This step helps us to increase variety of the rules.

1-3: finally, duplicated rules are eliminated from the final list.

\section{2) Rule selection phase}

For selecting best combination of rules from the available list of rules and also generation suitable weights corresponding to the fuzzy inference, following approach is proposed,

\section{a) Initial population:}

Three sets of chromosomes are needed; the first set presents candidate rules that are selected from the available list of rules. In this set each chromosome is an array with length equal to the number of total rules and each cell can be " 1 " or " 0 " that shows if the corresponding rule is selected for the fuzzy system or not. The first chromosome only contains ones therefore the related fuzzy systems using all of the available rules. Others chromosomes are made by random.

The second set of chromosomes presents weight w. Again this chromosome is also array with length equal to the number of available rules and each cell is equal to the importance of that rule, and it is a real number between 0 and 1 that generated randomly.

The final set of the chromosomes, represent weight $\mathrm{w}^{\wedge}$. This chromosome is an array with length equal to 4 . Each cell is the degree of importance of the related input. It can take a real number between 0 and 1 which generated by random.

\section{b) Fitness function:}

Fitness function for each chromosome is computed as the percentage of covered apnea/hypopnea events by the corresponding fuzzy system inference.

\section{c) Reproduction:}

Chromosomes are sorted by attention to their fitness function and best ones of them are selected for next generation. The number of this sets determined by percentage of reproduction.

\section{d) Crossover:}

First, two chromosomes selected randomly. Then mean of their fitness values stored as old mean, then two random number ind1 and ind2 are selected and two new chromosomes are created by "two point crossover" as Figure1 illustrated. Then if the mean value of fitness of the new chromosomes is better than the old mean, the new chromosomes will be selected for the next generation, and if, after specified number of try better pair of chromosomes cannot be found then the first pair is selected for the next generation.

\section{e) Mutation:}

One chromosome and one cell of that chromosome are selected by random and then value of that cell will be change to opposite value.

Note: same crossover and mutation is applied for weight $\mathrm{w}$, but weight $\mathrm{w}^{\prime}$ optimized by random search.

\section{f) Ending condition:}

Generating of new generations continued until specified number (Number of generation).

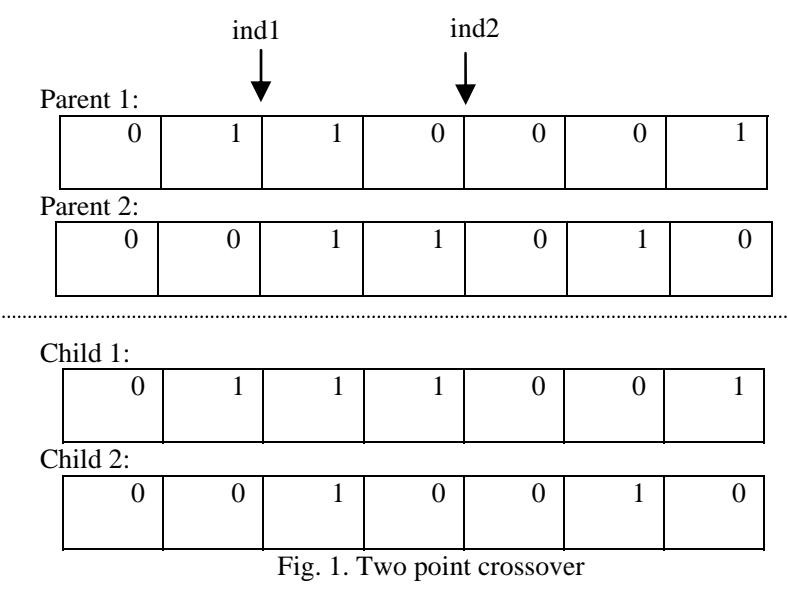

\section{VALIDATION OF RESUlTS}

Two samples with total duration of 75600 s and 959 apnea or hypopnea events in total are analyzed.

In the first phase 40 and 54 rules are generated respectively. Then, quarter of RUs of each sample is used for the learning phase with proposed genetic algorithms, and, 9 and 12 rules are selected respectively. As an example of the final rules please consider the following rule,

If Oxygen saturation fall is strong and Airflow fall is strong and Thoracic movement fall is very strong or strong then Status is event.

By using the obtained rules, RUs are labeled. Table IV shows the obtained results. It must be notice that the human expert evaluation was considered as a standard reference.

TABLE IV: OBTAINED RESULTS

\begin{tabular}{cccc}
\hline Sample & Duration & Number of events & Agreement (\%) \\
\hline$\# 1$ & $36000(\mathrm{~s})$ & 406 & $99 \%$ \\
$\# 2$ & $39600(\mathrm{~s})$ & 553 & $91 \%$ \\
\hline
\end{tabular}

\section{CONCLUSION}

In this paper an automated approach for detecting sleep apnea by fuzzy inference system is introduced. In this 
approach fuzzy rules and weights are generated by genetic algorithm. The proposed approach has three steps, in the first step, input biosignals are analyzed and events in each of them are determined. In the second step, the events are evaluated and RUs are constructed. In the third step, Genetic fuzzy approach is used for the final evaluating of RUs.

Based on the results, we can find that the proposed genetic fuzzy approach can be used for generating fuzzy rules for detecting sleep apnea/hypopnea events. But, for improving the accuracy for general samples, more study is needed. Also, we can use the same approach for classification of detected RUs as Apnea or Hypopnea in future works.

\section{REFERENCES}

[1] M. Klink and S. F. Quan, "Prevalence of reported sleep disturbances in a general adult-population and their relationship to obstractive airways diseases," Chest, vol. 91, pp. 540-546, Apr 1987.

[2] S. Chokroverty, C. Sudhansu, Md, Frcp, and Facp, "Approach to the Patient with Sleep Complaints," in Sleep Disorders Medicine (Third Edition) Philadelphia: W.B. Saunders, 2009, pp. 255-274.

[3] T. B. Young, "Epidemiology of daytime sleepiness: Definitions, symptomatology, and prevalence," Journal of Clinical Psychiatry, vol. 65, pp. 12-16, 2004.

[4] S. Chokroverty, C. Sudhansu, Md, Frcp, and Facp, "Sleep deprivation and sleepiness," in Sleep Disorders Medicine (Third Edition) Philadelphia: W.B. Saunders, 2009, pp. 22-28.

[5] E. M. Ball, R. D. Simon, A. A. Tall, M. B. Banks, G. NinoMurcia, and W. C. Dement, "Diagnosis and treatment of sleep apnea within the community - The Walla Walla project," Archives of Internal Medicine, vol. 157, pp. 419-424, Feb 1997.

[6] M. H. Kryger, L. Roos, K. Delaive, R. Walld, and J. Horrocks, "Utilization of health care services in patients with severe obstructive sleep apnea," Sleep, vol. 19, pp. S111-S116, 1996.

[7] J. R. Stradling and J. H. Crosby, "Relation between systemic hypertension and sleep hypoxemia or snoring- analysis in 748 men drawn from general-practice," British Medical Journal, vol. 300, pp. 75-78, Jan 1990.

[8] V. Hoffstein, "Snoring," in Principles and practice of sleep medicine, M. H. Kryger, T. Roth, and D. W. C., Eds. Philadelphia, PA: W.B.: Saunders, 2000, pp. 813-826.

[9] T. Penzel, J. McNames, P. de Chazal, B. Raymond, A. Murray, and G. Moody, "Systematic comparison of different algorithms for apnoea detection based on electrocardiogram recordings," Medical \& Biological Engineering \& Computing, vol. 40, pp. 402-407, Jul 2002.

[10] M. H. Kryger, "management of obstractive sleep-apnea," Clinics in Chest Medicine, vol. 13, pp. 481-492, Sep 1992.
[11] W. W. Flemons, D. Buysse, S. Redline, A. Pack, K. Strohl, J. Wheatley, T. Young, N. Douglas, P. Levy, W. McNicholas, J. Fleetham, D. White, W. Schmidt-Nowarra, D. Carley, J. Romaniuk, and F. Amer Acad Sleep Med Task, "Sleep-related breathing disorders in adults: Recommendations for syndrome definition and measurement techniques in clinical research," Sleep, vol. 22, pp. 667-689, Aug 1999.

[12] M. Cabrero-Canosa, E. Hernandez-Pereira, and V. Moret-Bonillo, "Intelligent diagnosis of sleep apnea syndrome," Ieee Engineering in Medicine and Biology Magazine, vol. 23, pp. 72-81, 2004.

[13] M. Cabrero-Canosa, M. Castro-Pereiro, M. Grana-Ramos, E. Hernandez-Pereira, V. Moret-Bonillo, M. Martin-Egana, and H. Verea-Hernando," An intelligent system for the detection and interpretation of sleep apneas," Expert Systems with Applications, vol. 24, pp. 335-349, 2003.

[14] P. de Chazal, C. Heneghan, E. Sheridan, R. Reilly, P. Nolan, and M. O'Malley, "Automated processing of the single-lead electrocardiogram for the detection of obstructive sleep apnoea," Ieee Transactions on Biomedical Engineering, vol. 50, pp. 686-696, 2003.

[15] H. Nazeran, A. Almas, K. Behbehani, J. Burk, and E. Lucas, "A fuzzy inference system for detection of obstructive sleep apnea," Proceedings of the 23rd Annual International Conference of the Ieee Engineering in Medicine and Biology Society, Vols 1-4, vol. 23, pp. 1645-1648, 2001.

[16] D. Alvarez-Estevez and V. Moret-Bonillo, "Fuzzy reasoning used to detect apneic events in the sleep apnea-hypopnea syndrome," Expert Systems with Applications, vol. 36, pp. 7778-7785, May 2009.

[17] J. Casillas and P. Martinez, "Consistent, complete and compact generation of DNF-type fuzzy rules by a Pittsburgh-style genetic algorithm," 2007 Ieee International Conference on Fuzzy Systems, vol. 1-4, pp. 1750-1755, 2007.

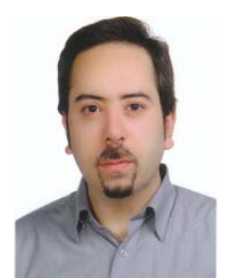

Yashar Maali received the Master Degree in Industrial Engineering. He is a $\mathrm{PhD}$ student in artificial intelligent in Faculty of Engineering and Information Technology, University of Technology, Sydney (UTS), Australia. His research interests include fields of decision making and artificial intelligent systems.

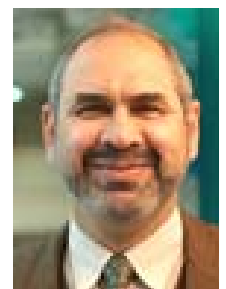

Adel Al-Jumaily received the Ph.D. Degree in electrical engineering (AI). He is Associate Professor in Faculty of Engineering and Information Technology, University of Technology, Sydney (UTS), Australia. His research interests include fields of biomechatronics, artificial intelligent systems, healthcare and biomedical engineering. 